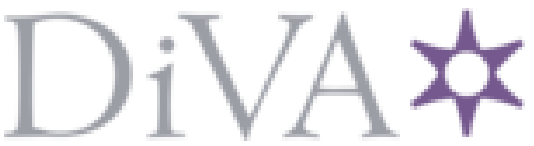

http://www.diva-portal.org

Postprint

This is the accepted version of a paper presented at 13th IEEE PowerTech 2019, 23-27 June, Milano, Italy.

Citation for the original published paper:

Herre, L., Dalton, J., Söder, L. (2019)

Optimal Day-Ahead Energy and Reserve Bidding Strategy of a Risk-Averse Electric Vehicle Aggregator in the Nordic Market

In: IEEE

https://doi.org/10.1109/PTC.2019.8810937

N.B. When citing this work, cite the original published paper.

Permanent link to this version:

http://urn.kb.se/resolve?urn=urn:nbn:se:kth:diva-254616 


\title{
Optimal Day-Ahead Energy and Regulation Self-Scheduling of a Risk-Averse Electric Vehicle Aggregator in the Nordic Market
}

\author{
Lars Herre, Student Member, IEEE, Jacob Dalton, and Lennart Söder, Senior Member, IEEE
}

\begin{abstract}
Electric vehicles (EV) can be considered as energy storage with availability, energy and capacity constraints that can provide flexibility to the power system in the form of balancing products when aggregated. In this paper, we develop a two-stage stochastic optimization problem that maximizes the profit of a risk-averse EV aggregator for bids on the day ahead in both energy and Frequency Containment Reserve (FCR) markets. Unidirectional charging is examined, while we take into account uncertainty from prices and vehicle availability. Case studies are carried out in different Nordic bidding areas based on historical EV charging data. We identify a strong temporal alignment of EV availability and high FCR-N prices. Results show that consumption is shifted largely towards early hours of the morning. When compared to a reference cost of charging case, up to $50 \%$ of the cost of charging can be recovered in Norway, and $100 \%$ in Sweden.
\end{abstract}

Index Terms-ancillary services, balancing markets, demand side management, electric vehicles.

\section{NOMENCLATURE}

\section{Indexes and Sets}

$\omega \in \Omega$ Index (set) of scenarios

$k \in \mathcal{K}^{t}$ Index (set) of sub-hourly time intervals within hour $\mathrm{t}$

$t \in \mathcal{T}$ Index (set) of hourly time intervals

$v \in \mathcal{V}$ Index (set) of electric vehicles

\section{Parameters}

$\alpha \quad$ Confidence level for risk evaluation $[0,1]$

$\beta \quad$ Risk aversion factor $[0,1]$

$\Delta \kappa \quad$ Sub hourly time interval in hours, 15 minutes $=0.25$.

$\eta_{v} \quad$ Efficiency of charging

$\lambda^{I P} \quad$ Imbalance Penalty $\left[\frac{\mathrm{NOK}}{\mathrm{MWh}}, \frac{\mathrm{SEK}}{\mathrm{MWh}}\right]$

$\lambda_{t}^{D A} \quad$ Day-ahead electricity price $\left[\frac{\mathrm{NOK}}{\mathrm{MWh}}, \frac{\mathrm{SEK}}{\mathrm{MWh}}\right]$

$\lambda_{t}^{D}$ Day-ahead FCR-D regulation price $\left[\frac{\mathrm{NOK}}{\mathrm{MW}}, \frac{\mathrm{SEK}}{\mathrm{MW}}\right]$

$\lambda_{t}^{N} \quad$ Day-ahead FCR-N regulation price $\left[\frac{\mathrm{NOK}}{\mathrm{MW}}, \frac{\mathrm{SEK}}{\mathrm{MW}}\right]$

$\lambda_{t, \omega}^{R T} \quad$ Real-time electricity price, i.e. Imbalance settlement (RPM price) $\left[\frac{\mathrm{NOK}}{\mathrm{MWh}}, \frac{\mathrm{SEK}}{\mathrm{MWh}}\right]$

$\bar{P}_{v} \quad$ Rated (maximal) charging power of vehicle $v[\mathrm{~kW}]$

$\bar{S}_{v}^{v} \quad$ Battery capacity of vehicle $v[\mathrm{kWh}]$

$\pi_{\omega} \quad$ Probability of scenario $\omega$

$A_{k, v, \omega}$ Binary parameter for vehicle availability which is 1 during the time interval $k \in\left[T_{v, \omega}^{a r r}, T_{v, \omega}^{d e p}\right]$

$R_{t}^{d c} \quad$ Dispatch to contract ratio, activation of FCR-D

$S_{v, \omega}^{a r r} \quad$ state of charge at arrival of vehicle $v[0,1]$

$S_{v, \omega}^{d e p} \quad$ state of charge at departure of vehicle $v[0,1]$

L. Herre, J.Dalton and L. Söder are with the Department of Electric Power \& Energy Systems, KTH Royal Institute of Technology, 10044 Stockholm, Sweden e-mail: $\{$ lherre,jdalton,lsod $\} @$ kth.se.
$T_{v, \omega}^{a r r} \quad$ Arrival time of vehicle $v$

$T_{v, \omega}^{d e p} \quad$ Departure time of vehicle $v$

$Z^{D} \quad$ Binary parameter that enforces FCR-D if 1

$Z^{N} \quad$ Binary parameter that enforces FCR-N if 1

Variables

$\iota \quad$ Scenario dependent auxiliary for CVaR

$\Pi_{\omega} \quad$ Profit in scenario $\omega$ [NOK or SEK]

$\zeta \quad$ Auxiliary variable for $\mathrm{CVaR}$

$e_{t, \omega}^{\Delta I} \quad$ Instructed deviation of real-time energy consumption

from day-ahead bid due to activation of $r_{t, \omega}^{N}$

$e_{t, \omega}^{\Delta U} \quad$ Uninstructed deviation of real-time energy consumption from day-ahead bid

$e_{t, \omega}^{\Delta} \quad$ Real-time energy bid [kWh]

$e_{t}^{D A} \quad$ Day-ahead energy bid [kWh/h]

$e_{k, \omega}^{R T}$ Total sub-hourly real-time energy consumption $[\mathrm{kWh} \cdot \Delta \kappa]$

$e_{k, v, \omega}^{R T} \quad$ Real-time energy consumption of vehicle $v[\mathrm{kWh} \cdot \Delta \kappa]$

$e_{t, \omega}^{R T}$ Total real-time energy consumption [kWh]

$p_{k, v, \omega} \quad$ Real-time power consumption of vehicle $v$ [kWh]

$r_{t}^{D} \quad$ Day-ahead FCR-D (down) regulation bid $\left[\frac{\mathrm{kW}}{0.1 \mathrm{~Hz}}\right]$

$r_{t}^{N} \quad$ Day-ahead FCR-N (symmetric) regulation bid $\left[\frac{\mathrm{kW}}{0.1 \mathrm{~Hz}}\right]$

$s_{k, v, \omega}$ state of charge of vehicle $v[0,1]$

CVaR Conditional Value at Risk [NOK or SEK]

\section{INTRODUCTION}

Due to the inherent, but as of yet imperfectly harnessed value in demand side management by electric vehicle (EV) aggregation, there has been a wealth of research carried out in this field. Most results show lower charging costs and increased aggregator profits through arbitraging energy prices by using EV flexibility. The literature can mainly be divided into bi-directional charging, also referred to as vehicle-to-grid (V2G), and uni-directional charging with lower impact on battery lifetime.

In [1] the authors derive an optimal bidding strategy for electric vehicle aggregators in the day-ahead, real-time and regulation markets. The authors use synthetic EV parameters and driving behaviour to model time of arrival, departure and state of charge at arrival uncertainty. Three different optimization problems of independent aggregators making day-ahead decisions in the wholesale and secondary reserve markets are presented in [2]. Synthetic EV data was created in order to determine so called "flexible periods" while the impact of forecast errors and uncertainty was considered through the comparison of results of perfect forecasts with a naive 
forecasting method. The authors build upon this study in [3] by developing an operational management $\&$ control model to minimize the difference between contracted and actual charging schedules. They find that adding this operational layer provides even more value with a $30-35 \%$ decrease in charging cost with respect to pure day-ahead energy optimization. The optimal scheduling behaviour of a risk-averse aggregator is modelled in [4]. A comparison between two scenarios; one where the aggregator has no control and another where dynamic load control is exercised, is used to evaluate the value of EV flexibility. A different method is exploited in [5], where chance constraints and the Markov inequality are used to create an efficient algorithm whose performance was evaluated against existing algorithms. Two thousand data points collected from smart chargers in British Columbia were extrapolated to mimic the charging sessions of a 1,000 vehicle fleet.

Although there have been a large number of studies examining the bidding behaviour of EV aggregators, to the authors' best knowledge they are reliant on the creation of synthetic EV fleet data and driving behaviour [2], [3], [6]-[11], or utilize a small first hand data sample and extrapolate to a larger synthetic sample [5], [12]. Furthermore, none of the above looked at the self-scheduling problem for combined bids in energy and balancing markets in the Nordic market.

In this paper, we formulate the self-scheduling problem of a risk averse EV aggregator in the Nordic market. We assume that the aggregator has real-time information and control over its fleet and aims to maximise profits on the day ahead. We model uncertainty from EV availability and real-time energy prices. The results are evaluated in terms of total expected profits and the value of EV flexibility is quantified. The contributions of this paper are the following:

- We formulate the two stage stochastic optimisation of a risk-averse EV aggregator that aims to maximise its expected profits on the day ahead, including (FCR-N \& FCR-D) frequency regulation.

- We use real historical vehicle availability and battery state data to conduct case studies of bidding area NO5 in Norway and SE3 in Sweden.

- The case study shows a considerable value in coordinated EV aggregation \& control in the Nordic market.

The rest of this paper is structured as follows. In Section II the problem setup is outlined including the relevant markets, the various arrangements of the model used, together with the assumptions. Section III portrays the mathematical formulation of the models. Section IV outlines the available data and results of a case study in Norway and Sweden. We discuss the results in Section V and conclude in Section VI.

\section{Problem Setup}

We want to explore the business case of an EV aggregator that has real-time control over its fleet and exploits this flexibility to participate in the energy and balancing markets. The aggregator's goal is to make optimal risk-averse dayahead decisions under uncertainty. While we ensure riskadversity and feasibility in the subsequent operation, the realtime control actions that would follow the day-ahead decisions are explicitly outside of the scope of this study. The aim is rather to give an estimation of potential profits for an aggregator, that will then have to weigh those against the cost of control systems and actions.

\section{A. Markets Considered}

This study is focused on the Nordic electricity markets, but can be used for most market setups that have a liberalized market for energy and frequency regulation services. Here, we assume that the aggregator can enter and place bids in the markets with the superscripts introduced below:

$D A$ : The day-ahead energy market closes at 12:00 CET D1 and is traded at the wholesale market Nordpool as a hourly product.

$R T$ : Real-time energy market. A one-price consumption imbalance settlement is used where the aggregator pays

$$
e_{t}^{D A} \cdot \lambda_{t}^{D A}-\left(e_{t}^{D A}-e_{t, \omega}^{R T}\right) \cdot \lambda_{t, \omega}^{R T}
$$

for energy delivery at hour $t$. In the Nordic market, $\lambda_{t, \omega}^{R T}$ corresponds to the price of the Regulating Power Market, that is announced ex-post after imbalance settlement.

$R$ : Primary frequency regulation $(\mathrm{FCR})^{1}$ is procured by the TSO up until 16:00 CET D-1.

$N$ : Frequency containment reserve in normal operation (FCR-N) $)^{2}$

$D$ : Frequency containment reserve in disturbed operation (FCR-D) was examined only for Sweden since the price for this product in Norway is negligible.

We include an imbalance penalty $\lambda^{I P}$ to account for uninstructed real-time deviations from the DA energy bids.

\section{B. Uncertainty}

Two main sources of uncertainty are considered: price uncertainty and availability due to driving behaviour.

- Price: Perfect price information is assumed for day-ahead $\left(\lambda_{t}^{D A}\right)$ and FCR prices $\left(\lambda_{t}^{N / D}\right)$ which is in line with the literature [1], [8], [16]. If the study is conducted for summer and winter prices separately, a clear diurnal pattern of FCR-N prices can be identified. Price uncertainty is reflected via daily real-time $\left(\lambda_{t, w}^{R T}\right)$ price trajectory scenarios $(\omega \in \Omega)$ based on historical market data.

- Availability: Driving behavior can vary between vehicle type and owner. Driving behaviour scenarios were sampled randomly from the pool of historical EV trips. The database was obtained from historical trips of Tesla vehicles.

\footnotetext{
${ }^{1}$ Note that FCR-N and FCR-D markets are analysed separately in this paper. In reality, an aggregator would be capable of entering both markets and may obtain higher revenues from simultaneously offering both FCR-N and FCR-D bids.

${ }^{2}$ The use of EVs in providing frequency containment reserves has been proven in field tests [13], [14] to satisfy the technical requirements of Nordic TSOs, with the observed response time of 5-6 seconds being well below the $63 \%$ in 60 seconds response mandated for FCR-N [15] for instance.
} 


\section{Risk Adversity - Conditional Value at Risk (CVaR)}

The mathematical formulation described in Section III accounts for risk adversity through the CVaR. The risk represented by CVaR can be described as the expected value of the profit of the $(1-\alpha)$-quantile of the profit distribution [17]. In other words, given a confidence interval $\alpha$ (here $90 \%$ ), the CVaR would return the average of all the bottom $(1-\alpha) \%$ of expected profits from the profit distribution. Therefore, by including $\mathrm{CVaR}$ in the objective function, the model is shifted from a risk-neutral to a risk-averse formulation with increasing $\beta$. Hereby, the sum of the expected profit $\mathbb{E}\left[\boldsymbol{\Pi}_{\omega}\right]$ and the bottom $(1-\alpha)$ percent of profits is maximized.

\section{Assumptions}

This model isolates the profits of the aggregator derived from participation in the wholesale electricity and reserve markets, from the income generated via retail contracts entered into with end consumers. The operational business and contractual details of the EV aggregator with its end consumers however, is outside the scope of this work. The contract offered to end consumers could include incentives such as price reduction of a form that remunerates the end consumer for transferring the control of the vehicle charging to the aggregator. The details of the end-consumer contract might influence charging patterns and consumer behavior.

With the presented problem formulation, the profits of the aggregator under uncertain price and charging profiles can be analysed irrespective of the business model of the aggregator and without the impacts that a specific customer contract type might have on charging patterns. Furthermore, we make the following assumptions:

- The aggregator is a price taker and thus has no effect on market prices.

- The aggregator is capable of dynamic load control in real time operation, i.e. it has the capability to remotely and continuously charge individual EVs.

- Each vehicle is assumed to only have one charging cycle per day available for control by the aggregator. This charging cycle is selected here as the single longest trip in each day. This assumption approximates the flexibility in a conservative way.

- The minimum bid size is always fulfilled. This assumption can be met in the Nordic context since Balance Responsible Parties are permitted to consolidate bids from various resources to meet minimum bid sizes [18].

- The real-time activation of primary FCR-N regulation has a zero mean character. This assumption is based on the fact that FCR-N is a symmetric product, aiming to maintain the frequency at $50 \mathrm{~Hz}$ and thereby having approximately equal up \& down regulation.

\section{Profit Maximization Problem}

The objective of the risk-averse aggregator is to maximize the expected profit over $x=\left\{e_{t, \omega}^{\Delta I}, e_{t, \omega}^{\Delta U}, e_{t, \omega}^{\Delta}, e_{t}^{D A}, e_{k, \omega}^{R T}\right.$, $\left.e_{k, v, \omega}^{R T}, e_{t, \omega}^{R T}, p_{k, v, \omega}, r_{t}^{N}, r_{t}^{D}, s_{k, v, \omega}, \mathrm{CVaR}, \iota, \zeta\right\}$ considering the Conditional Value at Risk

$$
\max _{x} \cdot(1-\beta) \cdot \mathbb{E}\left[\boldsymbol{\Pi}_{\omega}\right]+\beta \cdot \mathrm{CVaR}
$$

subject to (3),(4) to (8) where

$$
\mathbb{E}\left[\boldsymbol{\Pi}_{\omega}\right]=\Pi^{R}-\Pi^{D A}-\sum_{\omega \in \Omega} \pi_{\omega}\left(\Pi_{\omega}^{R T}+\Pi_{\omega}^{I P}\right) .
$$

The expected profit (3) is composed of the day-ahead income (4a) from accepted frequency containment reserve bids, the day-ahead cost of energy in (4b), the expected cost (or revenue) from the purchase (or sale) of energy in real-time, represented by (4c), and finally the penalty due to deviation, in the form of a consumption imbalance fee in (4d). Note that in this study, the term "real-time" refers to the imbalance settlement and therefore is associated with the RPM price.

$$
\begin{aligned}
\Pi^{R} & =\sum_{t \in T} Z^{N} r_{t}^{N} \lambda_{t}^{N}+Z^{D} r_{t}^{D} \lambda_{t}^{D} & \\
\Pi^{D A} & =\sum_{t \in T} e_{t}^{D A} \lambda_{t}^{D A} & \\
\Pi_{\omega}^{R T} & =\sum_{t \in \mathcal{T}} e_{t, \omega}^{\Delta} \cdot \lambda_{t, \omega}^{R T} & \forall \omega \\
\Pi_{\omega}^{I P} & \geq \sum_{t \in T} e_{t, \omega}^{\Delta} \cdot \lambda^{I P} & \forall \omega \\
\Pi_{\omega}^{I P} & \geq-\sum_{t \in T} e_{t, \omega}^{\Delta} \cdot \lambda^{I P} & \forall \omega
\end{aligned}
$$

Equation (5a) gives the volume of energy that is purchased or sold in real-time and is equivalent to the deviation between real-time energy consumption and the day-ahead bid. (5b) and (5c) outline the aggregated hourly real-time consumption as the sum of all sub hourly $(k)$ real-time consumption of vehicles in set $\mathcal{V}$. Equation (5d) gives an approximation of real-time energy consumption through the trapezoidal rule of $p_{k, v, \omega}$ at $k$ and $k+1$, while (5e) constrains $p_{k, v, \omega}$ to be less than or equal to the rated charge power when the vehicle is available.

$$
\begin{array}{rlrl}
e_{t, \omega}^{\Delta} & =e_{t, \omega}^{R T}-e_{t}^{D A} & & \forall t, \omega \\
e_{t, \omega}^{R T} & =\sum_{k \in \mathcal{K}^{t}} e_{k, \omega}^{R T} & & \forall t, \omega \\
e_{k, \omega}^{R T} & =\sum_{v \in \mathcal{V}} e_{k, v, \omega}^{R T} & & \forall k, \omega \\
e_{k, v, \omega}^{R T} & \leq \frac{p_{k, v, \omega}+p_{k+1, v, \omega}}{2} \Delta \kappa & \forall k, \omega \\
p_{k, v, \omega} & \leq A_{k, v, \omega} \cdot \bar{P}_{v} & \forall k, v, \omega
\end{array}
$$

The state of charge (SOC) between time steps $k$ is recursively defined by (6a) and constrained in (6b). The maximum battery capacity $\bar{S}_{v}$ is used to normalize real time charging with respect to the SOC. Equations (6c) and (6d) define the $\mathrm{SOC}$ at arrival $\left(T_{v, \omega}^{a r r}\right)$ and departure $\left(T_{v, \omega}^{d e p}\right)$ respectively.

$$
\begin{aligned}
s_{k+1, v, \omega} & =s_{k, v, \omega}+\eta_{v} \cdot \frac{e_{k, v, \omega}^{R T}}{\bar{S}_{v}} & & \forall k, v, \omega \\
0 & \leq s_{k, v, \omega} \leq 1 & & \forall k, v, \omega \\
s_{T_{v, \omega}^{d e p}, v, \omega} & \geq S_{v, \omega}^{d e p} & & \forall v, \omega \\
s_{T_{v, \omega}^{a r r}, v, \omega} & =S_{v, \omega}^{a r r} & & \forall v, \omega
\end{aligned}
$$


The Conditional Value at Risk (CVaR) is reflected in (7a) to $(7 \mathrm{c})$. The level of risk aversion is determined by the parameter $\beta$ in the objective function (2) as described in Section II-C.

$$
\begin{aligned}
\mathrm{CVaR} & =\zeta-\frac{1}{(1-\alpha)} \sum_{\omega \in \Omega} \pi_{\omega} \iota_{\omega} \\
\zeta-\mathbf{\Pi}_{\omega} & \leq \iota_{\omega} \\
\iota_{\omega} & \geq 0
\end{aligned}
$$$$
\forall \omega
$$$$
\forall \omega
$$

Here, we assume that the aggregator would bid in only one of the FCR markets, i.e. $Z^{N}+Z^{D} \leq 1$. When the FCR-N market is considered, (8a) and (8b) are enforced. The maximum upwards regulation capacity is determined by (8a), while the maximum downwards regulation capacity is calculated in (8b). When the FCR-D market is considered, (8c) and (8d) are enforced. When $Z^{N}=Z^{D}=0$, then $\Pi^{R}=0$ and the aggregator would only arbitrage energy and exploit price difference between the DA and RT energy prices.

$$
\begin{aligned}
r_{t, \omega}^{N} & \leq e_{t, \omega}^{R T} \cdot Z^{N} & \forall t, \omega \\
r_{t, \omega}^{N} & \leq \sum_{v \in \mathcal{V}} A_{k, v, \omega} \bar{P}_{i}-e_{t, \omega}^{R T} & \forall t, k \in \kappa_{t}, \omega \\
e_{t, \omega}^{\Delta} & =e_{t, \omega}^{I} \cdot Z^{D}+e_{t, \omega}^{\Delta U} & \forall t, \omega \\
e_{t, \omega}^{\Delta I} \cdot Z^{D} & =R_{t}^{d c} \cdot r_{t}^{D} & \forall t, \omega
\end{aligned}
$$

FCR-N is assumed approximately symmetrical while FCR-D is only up regulating. Up regulation of generation corresponds to down regulation of EV consumption. Similar to the model in [1], the activation of the bid is considered here via a dispatchto-contract ratio $R_{t}^{d c}$. This parameter provides the proportion of submitted FCR-D bids that will be activated in real-time. An activation of FCR-D however, would result in a deviation from the day-ahead energy bid and resulting imbalance penalty. Therefore, we split the imbalance into instructed $e_{t, \omega}^{\Delta I}$ and uninstructed $e_{t, \omega}^{\Delta U}$ deviation where only the latter is penalized. The profit maximization of the aggregator can be formulated as a linear program (LP) given the assumptions in Section II-D.

Market Participation Cases: Based on the general formulation of (3), we can modify the problem to study different cases of the aggregator's involvement. To this end, the total profits of the aggregator are labeled with the respective superscripts in the rest of this paper.

$\mathcal{R}$ : The reference case: The aggregator acts as a retailer and has no control, i.e. conducts immediate charging after each trip until fully charged. To this end, the availability parameter $A_{k, v, \omega}$ is modified $\left(Z^{D}=Z^{N}=0\right)$.

$\mathcal{A}$ : Energy Arbitrage: The aggregator only participates in the energy markets, i.e. $\Pi^{R}=0\left(Z^{D}=Z^{N}=0\right)$.

$\mathcal{N}$ : FCR-N \& Energy Arbitrage: The aggregator participates in the energy markets and FCR-N market with capacity offer $R_{t}$, i.e. $\Pi^{R}=\sum_{t \in T} r_{t}^{N} \cdot \lambda_{t}^{N}\left(Z^{N}=1\right)$.

$\mathcal{D}$ : FCR-D \& Energy Arbitrage: The aggregator participates in the energy markets and FCR-D market with capacity offer $R_{t}$, i.e. $\Pi^{R}=\sum_{t \in T} r_{t}^{D} \cdot \lambda_{t}^{D}\left(Z^{D}=1\right)$.

\section{CAse Study}

\section{A. Data}

Vehicle Data: We use real historical data of vehicle availability as well as the actual charging power, battery size, $\mathrm{SOC}$ at arrival and departure. We only model uni-directional charging (no V2G) and assume a constant charging efficiency of $\eta_{v}=0.9 \forall v$.

Market Price Data: The day-ahead $\left(\lambda_{t}^{D A}\right)$ and real-time price $\left(\lambda_{t, \omega}^{R T}\right)$ are publicly available on Nord Pool's website [19]. Meanwhile, the FCR-N \& FCR-D regulation prices $\left(\lambda_{t}^{N}, \lambda_{t}^{D}\right)$ are available from the TSO's (Statnett and Svenska krafnät) data portals for Norwegian and Swedish market data respectively. For each season (S: summer, W: winter), the hourly mean of historical prices was used for both the dayahead energy and FCR prices where perfect price information was assumed. The motivation for this assumption is that the daily FCR-N price trajectories on weekdays shows very similar magnitudes throughout a given season. An illustration

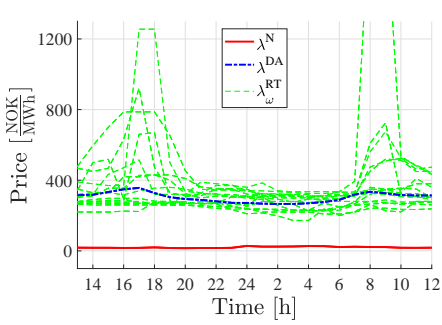

(a) NO5-W: Prices

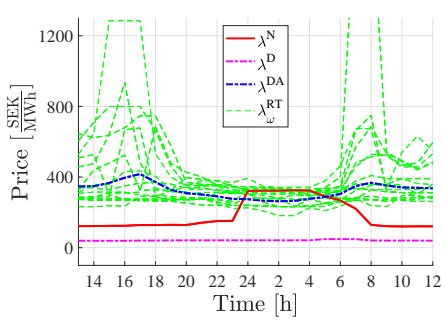

(c) SE3-W: Prices

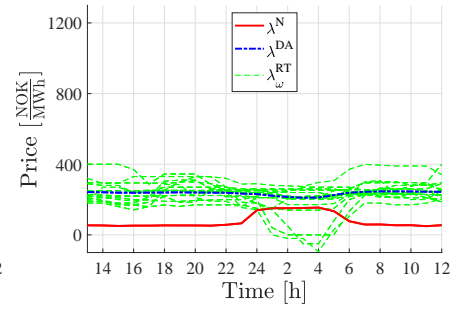

(b) NO5-S: Prices

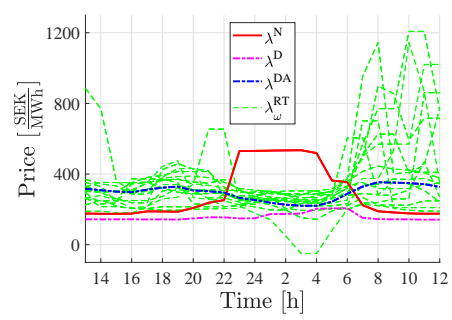

(d) SE3-S: Prices
Fig. 1. Prices in NO5 and SE3: Day-ahead energy $\left(\lambda^{D A}\right), \operatorname{FCR}-\mathrm{N}\left(\lambda^{N}\right)$, FCR-D $\left(\lambda^{D}\right)$, and 15 scenarios of RT energy prices $\left(\lambda_{\omega}^{R T}\right)$.

of characteristic market data in bidding areas NO5 and SE3 is given in Fig. 1. It displays the day-ahead and FCR-N prices in bold, while real-time price scenarios are indicated by thin lines. The 15 real-time price scenarios were found based on a forward selection technique [20]. Note that the FCR-N price significantly increases during the night hours, and even more so in summer.

\section{B. Results}

The breakdown of results over a 24 hour period in summer and winter is outlined in Table I with market prices based on bidding area NO5 and SE3. The aggregator's expected profit is the sum of the day-ahead and expected real-time energy cost and the return from provision of FCR-N. It is observed that the expected real-time energy cost is positive in summer and is therefore representing a return from arbitrage between the 
TABLE I

EXPECTED DAILY PRofits OF THE 806 VEHICLE FLEET IN NO5 AND SE3

\begin{tabular}{lrrrrrr}
\hline & \multicolumn{2}{c}{$\mathcal{N}[\mathrm{NOK}]$} & \multicolumn{2}{c}{$\mathcal{N}[\mathrm{SEK}]$} & \multicolumn{2}{c}{$\mathcal{D}[\mathrm{SEK}]$} \\
& NO5-W & NO5-S & SE3-W & SE3-S & SE3-W & SE3-S \\
\hline Regulation & 191.3 & $1,042.5$ & $2,326.0$ & $3,837.2$ & 339.5 & $1,501.7$ \\
DA Energy & $-2,179.1$ & $-1,969.4$ & $-2,391.4$ & $-2,223.2$ & $-2,361.7$ & $-2,210.0$ \\
RT Energy & -52.9 & 106.8 & 40.4 & 142.4 & 36.4 & 115.5 \\
CVaR & $-2,235.6$ & $-9,23.8$ & -164.1 & $1,645.2$ & $-2,131.2$ & -700.9 \\
\hline Total Profit & $\mathbf{- 2 , 1 5 4 . 5}$ & $\mathbf{- 8 7 3 . 3}$ & $\mathbf{- 1 0 4 . 1}$ & $\mathbf{1 , 6 7 9 . 4}$ & $\mathbf{- 2 , 0 8 5 . 3}$ & $\mathbf{- 6 6 6 . 5}$
\end{tabular}

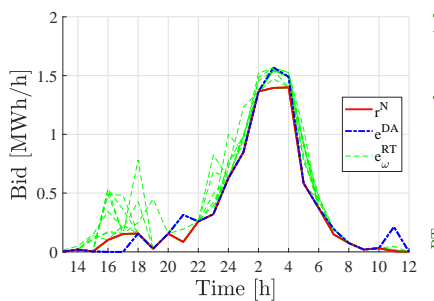

(a) NO5-S: Bids

(c) SE3-S: Bids

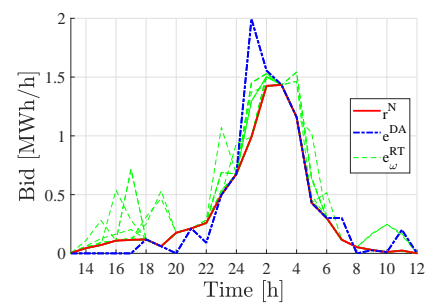

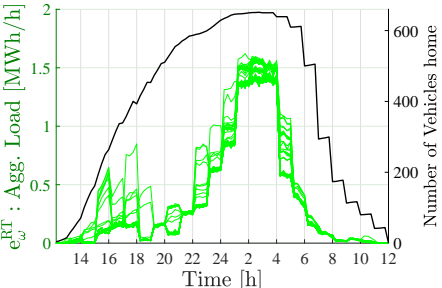

(b) NO5-S: Load

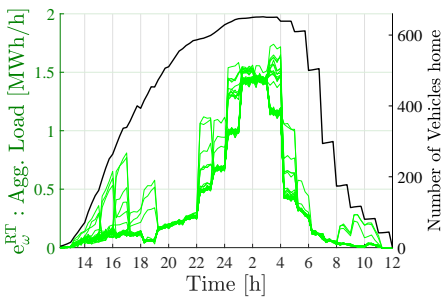

(d) SE3-S: Load
Fig. 2. Results for summer NO5-S and SE3-S case studies. The left hand figures show the optimal day-ahead bids as well as scenario dependent realtime energy consumption. The right hand figures show the number of available EVs as well as the scenario dependent utilized aggregated load from the pool of available EVs.

day-ahead and real-time markets. The expected RT energy cost is positive in many cases, indicating a return through the sale of energy in real-time. In SE3 in summer, with a return from FCR-N provision of 3,837 SEK, the entire cost of charging is recovered and even surpassed. Hence the expected total profit is positive in this case. The reason this value is significantly greater when compared to the results from NO5, is almost entirely attributable to higher FCR-N prices in Sweden, c.f. Fig. 1. The expected return from providing primary regulation covers almost $55 \%$ of the charging cost. The lower regulation return in winter is attributed to lower FCR-N prices in the winter period, c.f. Figs. 1a and $1 \mathrm{~b}$.

The aggregated load curve of the EV fleet is visualized in Fig. $2 b$ with the green lines portraying the aggregate load resulting from EV availability in various scenarios. The black line displays the mean number of vehicles that are "home \& connected" at each 15 minute interval, showing the largest drop as drivers leave for work between $7 \mathrm{am}$ and $8 \mathrm{am}$. It can be observed that charging is shifted to the hours of higher FCR-N prices between $12 \mathrm{am}-5 \mathrm{am}$, c.f. Fig. 1b, in order to maximize return. Meanwhile, the optimized day-ahead energy $\left(e^{D A}\right)$ and FCR-N regulation bids $\left(r^{N}\right)$ are shown in Fig. 2a, together with the scenarios of real-time consumption. Energy arbitrage is carried out where the day-ahead energy bid varies from the real-time energy consumption. This is clearly illustrated at $11 \mathrm{am}$ for instance, where the optimal schedule is to buy a volume of DA energy in excess of the real-time consumption, in order to sell in the RT market at a higher price.

For market participation case $\mathcal{D}$, we use a conservative approach by selecting the $99^{\text {th }}$ percentile day with the highest $1 \%$ of recorded dispatch-to-contract ratio $R_{t}^{d c}$ over a full day. Selected percentile days are shown in Fig. 3. The significance

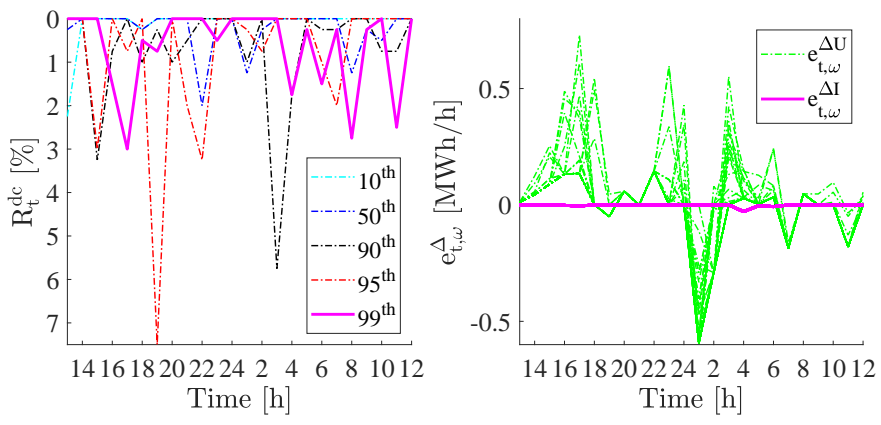

Fig. 3. Percentile days of dispatch-to-contract ratio $R_{t}^{d c}$ (left), instructed $\left(e_{t, \omega}^{\Delta I}\right)$ and un-instructed deviation $\left(e_{t, \omega}^{\Delta U}\right)$ in SE3-S (right).

of instructed deviation (where parts of FCR-D reserves are activated by the TSO) is low compared to the un-instructed deviation stemming from energy arbitrage between day-ahead and real-time markets. This means that there is more value in energy arbitrage than in providing FCR-D capacity, despite the penalty for un-instructed deviation.

\section{Value of Flexibility}

Let $\mathcal{R}$ be the reference case of uncontrolled charging (immediate charging after each trip) and $\{\mathcal{A}, \mathcal{N}, \mathcal{D}\} \in \mathcal{X}$ be the set of alternative market participation cases. The absolute $(\Phi)$ and relative $(\varphi)$ value of flexibility is defined analogous to [4] in (9) with respect to the reference of immediate charging.

$$
\begin{array}{rlr}
\Phi^{\mathcal{X}} & =\mathbb{E}\left[\boldsymbol{\Pi}_{\omega}^{\mathcal{X}}\right]-\mathbb{E}\left[\boldsymbol{\Pi}_{\omega}^{\mathcal{R}}\right] & {[\mathrm{NOK}, \mathrm{SEK}]} \\
\varphi^{\mathcal{X}} & =\frac{\mathbb{E}\left[\boldsymbol{\Pi}_{\omega}^{\mathcal{X}}\right]-\mathbb{E}\left[\boldsymbol{\Pi}_{\omega}^{\mathcal{R}}\right]}{\mathbb{E}\left[\boldsymbol{\Pi}_{\omega}^{\mathcal{R}}\right]} & {[\%]}
\end{array}
$$

Table II summarizes the absolute and relative value of flexibility of the entire fleet in SE3 and NO5 for the market participation cases $\mathcal{A}, \mathcal{N}, \mathcal{D}$. The value of flexibility of an

TABLE II

VAlue of Flexibility of THE 806 EV FLEET PER DAY, ${ }^{*}$ NOK, ${ }^{+}$SEK

\begin{tabular}{lrrrrr}
\hline & & NO5-W $^{*}$ & NO5-S $^{*}$ & SE3-W $^{+}$ & SE3-S $^{+}$ \\
\hline $\mathcal{R}$ : immediate charging & & $-2,537.13$ & $-2,021.51$ & $-2,562.05$ & $-2,441.15$ \\
$\mathcal{A}$ : energy arbitrage & $\Phi^{\mathcal{A}}$ & 224.89 & 200.65 & 205.23 & 466.68 \\
& $\varphi^{\mathcal{A}}$ & $9 \%$ & $10 \%$ & $8 \%$ & $19 \%$ \\
$\mathcal{N}$ : arbitrage + FCR-N & $\Phi^{\mathcal{N}}$ & 382.64 & $1,148.21$ & $2,457.97$ & $4,120.50$ \\
& $\varphi^{\mathcal{N}}$ & $15 \%$ & $57 \%$ & $96 \%$ & $196 \%$ \\
$\mathcal{D}$ : arbitrage + FCR-D & $\Phi^{\mathcal{D}}$ & & & 476.72 & $1,774.68$ \\
& $\varphi^{\mathcal{D}}$ & & & $19 \%$ & $73 \%$ \\
\hline
\end{tabular}

average vehicle per month is visualized in Fig. 4. Note that the value of flexibility changes significantly with the market participation cases and is generally higher in Sweden, and in summer. 


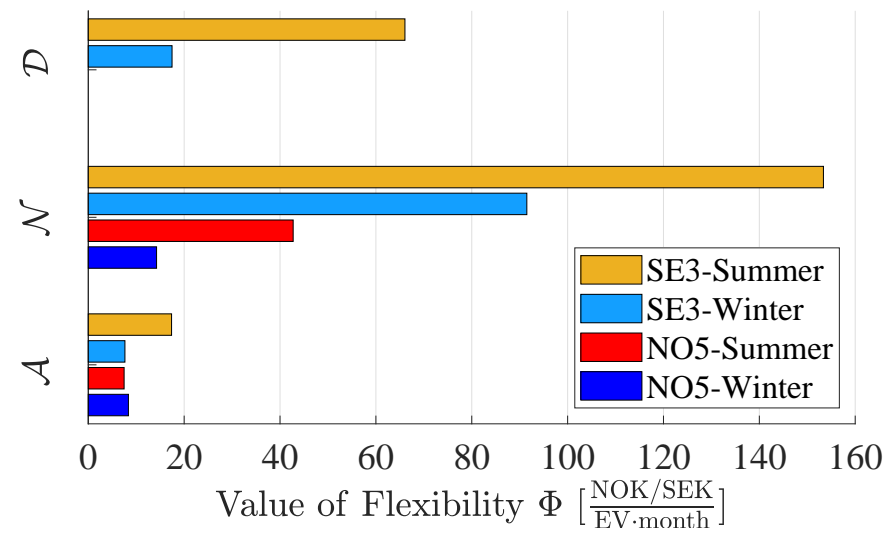

Fig. 4. Value of an average EV per month in the 806 vehicle fleet in various market participation cases and seasons in NO5, SE3.

\section{Discussion}

The results were obtained for summer and winter seasons separately by using the hourly mean DA energy and FCR($\mathrm{N} \&$-D) prices. Assuming return from regulation across the entire year is the mean of the return in winter and summer periods, these results can be extrapolated to an average yearly revenue per vehicle. This average yearly revenue from providing energy arbitrage and FCR-N $(\mathcal{N})$ amounts to 342 NOK (NO5) and 1,470 SEK (SE3) per vehicle respectively. With this market participation, an EV can essentially be charged "for free" in Sweden by recovering 96\% (winter) to $196 \%$ (summer) of the charging cost in energy and FCR-N markets. Note that these values do not consider other revenue streams, such as end user retail contracts which are dependent on the business model of the aggregator.

One flexibility limitation is that $50 \%$ of the home chargers in the examined EV fleet have capacities $\bar{P}_{v}$ of only 2 o 3 $\mathrm{kW}$, which means that an empty $100 \mathrm{kWh}$ Tesla vehicle would require more than 24 hours to be fully charged. Hence, it could be a significant value proposition to an end-user to offer an upgrade to a high-power home charging unit. A Tesla wall connector for instance, has a unit price of 5,200 SEK [21] (with a capacity of up to $16.5 \mathrm{~kW}$, excluding installation labor). This cost could be recovered in Sweden by an average vehicle within 3.5 years.

\section{CONCLUSION}

A two-stage stochastic optimization model was developed to maximise the expected profits of a risk-averse electric vehicle (EV) aggregator by an optimal day ahead schedule. The model was used to quantify the value from aggregating and controlling a fleet of EVs in the Nordic market. Case studies were carried out using real historical fleet data. Results showed moderate revenue from participation in the FCR-N market in Norway but greater revenues in Sweden stemming from considerably higher regulation prices. The expected profit of an EV aggregator from energy arbitrage and FCR-N provision is approximately $342 \mathrm{NOK}$ in Norway and 1,470 SEK in Sweden per vehicle per year. Compared to a reference cost of uncontrolled charging, up to $50 \%$ of the cost can be recovered in Norway, while the entire charging cost can be recovered in
Sweden. These results make strides in confirming the existence of significant value in exploiting the inherent flexibility of electric vehicle charging in the Nordic energy and frequency regulation markets.

\section{REFERENCES}

[1] S. I. Vagropoulos and A. G. Bakirtzis, "Optimal bidding strategy for electric vehicle aggregators in electricity markets," IEEE Transactions on Power Systems, vol. 28, no. 4, pp. 4031-4041, 2013.

[2] R. J. Bessa, M. A. Matos, F. J. Soares, and J. A. Lopes, "Optimized bidding of a EV aggregation agent in the electricity market," IEEE Transactions on Smart Grid, vol. 3, no. 1, pp. 443-452, 2012.

[3] R. J. Bessa and M. A. Matos, "Optimization models for an EV aggregator selling secondary reserve in the electricity market," Electric Power Systems Research, vol. 106, pp. 36-50, 2014.

[4] I. Momber, A. Siddiqui, T. G. S. Roman, and L. Soder, "Risk averse scheduling by a PEV aggregator under uncertainty," IEEE Transactions on Power Systems, vol. 30, no. 2, pp. 882-891, 2015.

[5] E. Yao, V. W. Wong, and R. Schober, "Risk-Averse forward contract for electric vehicle frequency regulation service," 2015 IEEE International Conference on Smart Grid Communications, pp. 750-755, 2016.

[6] M. Alipour, B. Mohammadi-Ivatloo, M. Moradi-Dalvand, and K. Zare, "Stochastic scheduling of aggregators of plug-in electric vehicles for participation in energy and ancillary service markets," Energy, vol. 118, pp. 1168-1179, 2017.

[7] T. W. Hoogvliet, G. B. Litjens, and W. G. van Sark, "Provision of regulating- and reserve power by electric vehicle owners in the Dutch market," Applied Energy, vol. 190, pp. 1008-1019, 2017.

[8] M. Shafie-Khah, M. P. Moghaddam, M. K. Sheikh-El-Eslami, and J. P. Catalão, "Optimised performance of a plug-in electric vehicle aggregator in energy and reserve markets," Energy Conversion and Management, vol. 97, pp. 393-408, 2015.

[9] M. Shafie-khah, N. Mahmoudi, P. Siano, T. K. Saha, and J. P. Catalao, "A Comprehensive Model to Integrate Emerging Resources from Supply and Demand Sides," IEEE Transactions on Smart Grid, vol. 3053, no. c, pp. 1-13, 2017.

[10] E. Sortomme and M. A. El-Sharkawi, "Optimal charging strategies for unidirectional vehicle-to-grid,' IEEE Transactions on Smart Grid, vol. 2, no. 1 , pp. 119-126, 2011 .

[11] — "Optimal scheduling of vehicle-to-grid energy and ancillary services," IEEE Transactions on Smart Grid, vol. 3, no. 1, pp. 351-359, 2012.

[12] B. Škugor and J. Deur, "Dynamic programming-based optimisation of charging an electric vehicle fleet system represented by an aggregate battery model," Energy, vol. 92, pp. 456-465, 2015.

[13] S. Martinenas, M. Marinelli, P. B. Andersen, and C. Traholt, "Implementation and demonstration of grid frequency support by $\mathrm{V} 2 \mathrm{G}$ enabled electric vehicle," in 2014 49th International Universities Power Engineering Conference (UPEC). IEEE, sep 2014, pp. 1-6.

[14] M. Marinelli, S. Martinenas, K. Knezović, and P. B. Andersen, "Validating a centralized approach to primary frequency control with seriesproduced electric vehicles," Advances in Life Course Research, vol. 7, pp. 63-73, 2016.

[15] Expektra, "Summary and Description of the different rules of the Markets in Sweden.” Expektra, Danderyd, Tech. Rep. 556818, 2017.

[16] A. Van Der Veen and M. Kaisers, "Computing the value of flexibility in electricity retail, ahead and balancing markets," IEEE PES Innovative Smart Grid Technologies Conference Europe, 2017.

[17] L. Barroso and A. Conejo, Decision making under uncertainty in electricity markets. Springer US, 2006.

[18] Svenska Kraftnät, "Slutrapport pilotprojekt Flexibla hushåll," Svenska Kraftnät, Sundbyberg, Tech. Rep. Svk 2016/1688, 2017.

[19] Nord Pool AS, "Nordpoolspot - Historical Market Data." [Online]. Available: http://www.nordpoolspot.com/historical-market-data/

[20] H. Heitsch and W. Römisch, "Scenario reduction algorithms in stochastic programming," Computational Optimization and Applications, vol. 24, no. 2, pp. 187-206, Feb 2003.

[21] Tesla Norway, "Installasjon av hjemmelading (in Norwegian)." [Online]. Available: https://www.tesla.com/no_NO/support/home-charginginstallation 